\title{
A 43 year old male patient presenting with intractable diarrhoea
}

N. Kavita, MBBS, MD, DMRD.

The Nuclear Medicine Unit, The Apollo-Gleneagles Hospital, Hyderabad, India.

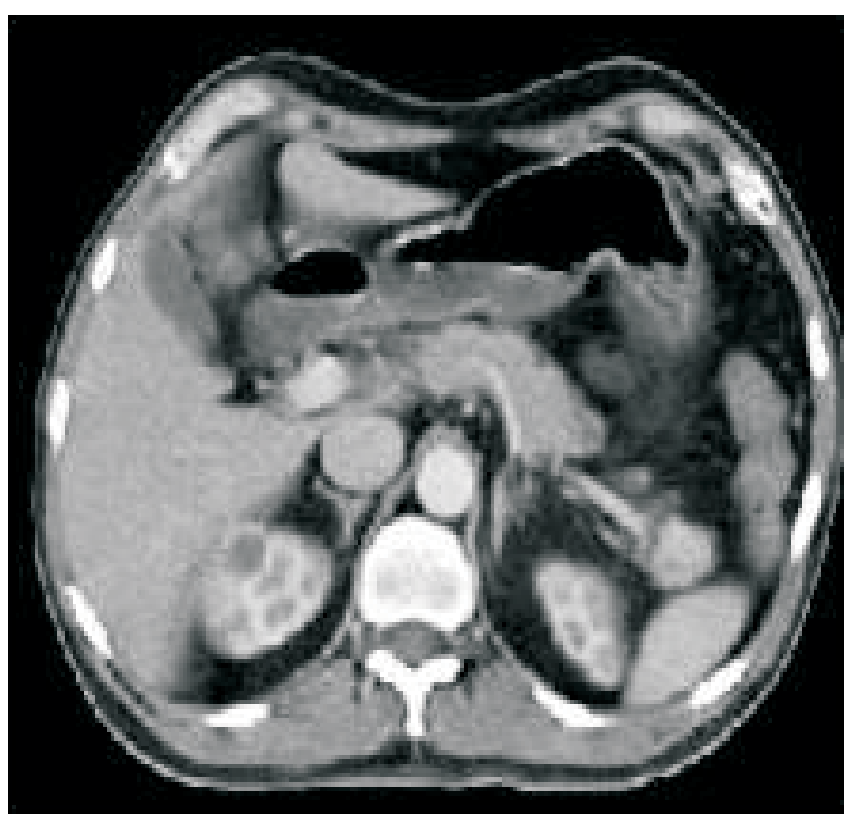

Figure A

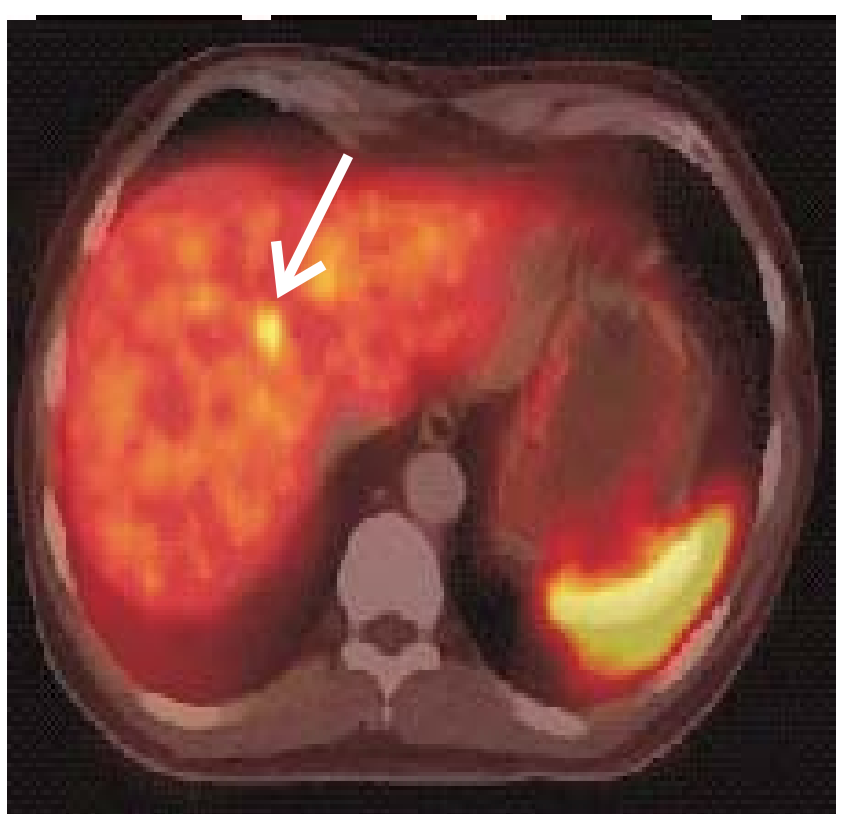

Figure B

A 43 year old man presented with intractable diarrhoea three years after surgery for parathyroid adenomas. Analysis of stool, upper and lower intestinal endoscopy, including capsule enteroscopy, was reported normal. Gastrin levels in serum were elevated. Computerised tomography (CT) revealed an enhancing mass in the tail of the pancreas (Figure A).

1. What does Figure B represent?

2. Which syndrome is this condition a part of?
Find the answers on page $\mathbf{5 0}$

Correspondence: Dr. N.Kavita, Head of the Department of Nuclear Medicine,

The Apollo-Gleneagles PET-CT Centre, Hyderabad, India.

Email: d_kavithareddy@rediffmail.com

The Sri Lanka Journal of Surgery $2011 ; 29(1): 37$. 